\title{
A novel extension of GS4-1 time integrator to fluid dynamics type non-linear problems with illustrations to Burgers' equation
}

\begin{abstract}
Purpose - The purpose of this paper is to describe how a generalized single-system-singlesolve (GS4-1) computational framework, previously developed for linear first order transient systems, can be properly extended for use in nonlinear counterparts, with particular applications to time dependent Burgers' equation, which is well-known to serve as a simplified model of fluid dynamics, for illustrations of the essential concepts. Design/methodology/approach - The framework permits, for a very general family of time integrators where traditional methods are a subset, much needed desirable features including second order time accuracy, robustness and unconditional stability, zero-order overshoot behavior, and additionally, a selective control of high frequency damping for both the primary variable and its time derivative. The latter, which is a new, key desirable feature not available in past/existing methods to-date, allows for different amounts of high frequency damping for both the primary variable and its time derivative to ensure physically accurate solutions of these variables. This is in contrast to having only limited control of these numerical dampings, often indiscriminately, as in some past developments which can lead to numerical instabilities in the time derivative variable. The extension of the framework to nonlinear problems, as described in this paper, is achieved via the use of a normalized time weighted residual approach, which naturally allows the time discretization of the transient nonlinear systems as being the natural extensions of the linear systems. Findings - The primary aim is also to demonstrate the advantage of the selective control feature inherit in the present numerical methodologies for these nonlinear first order transient systems as in the linear counterparts. Originality/value - The authors wish to tackle the challenges to further enable extensions to nonlinear first order transient systems that frequently arise in fluid dynamics problems; this is the focus of this paper. The primary wish is to demonstrate the ability of the GS4-1 framework for nonlinear first order transient systems as seen in the linear transient counterparts; while on one hand the authors show that an equal amount of high frequency damping (i.e.p $\infty$ ) leads to non-physical instability in the time derivative variable for a minimal damping required to obtain acceptable solution of the primary variable, on the other hand, the authors particularly demonstrate how this instability can be easily tuned off via the selective control feature (i.e.p $\infty \mathrm{s}$ ) offered by the developed framework; thereby, demonstrating its robustness and superiority.
\end{abstract}

Keyword: $\quad$ Time integration; Transient analysis; Nonlinear problems 